\title{
Pharmacokinetic/pharmacodynamic study of
}

\section{posaconazole delayed-release tablet in a patient with coexisting invasive aspergillosis and mucormycosis}

This article was published in the following Dove Press journal:

Therapeutics and Clinical Risk Management

\author{
Pannee Leelawattanachai ${ }^{1,2}$ \\ Preecha Montakantikul' \\ Wichit Nosoongnoen' \\ Methee Chayakulkeeree ${ }^{3}$ \\ 'Division of Clinical Pharmacy, \\ Department of Pharmacy, Faculty of \\ Pharmacy, Mahidol University, Bangkok, \\ Thailand; ${ }^{2}$ Department of Pharmacy, \\ Faculty of Medicine Vajira Hospital, \\ Navamindradhiraj University, Bangkok, \\ Thailand; ${ }^{3}$ Division of Infectious Diseases \\ and Tropical Medicine, Department of \\ Medicine, Faculty of Medicine Siriraj \\ Hospital, Mahidol University, Bangkok, \\ Thailand
}

Correspondence: Methee Chayakulkeeree Division of Infectious Diseases and Tropical Medicine, Department of Medicine, Faculty of Medicine Siriraj Hospital, Mahidol University, 2 Wanglang Road, Bangkokoi, Bangkok 10700, Thailand

Tel +6624199462

Fax +6624197783

Email methee.cha@mahidol.ac.th

\begin{abstract}
Limited information exists regarding the optimal dose of posaconazole delayedrelease tablet for the treatment of invasive mold infection. Here, we report the case of a previously healthy 44-year-old Thai man who developed coexisting invasive pulmonary aspergillosis and mucormycosis following a car accident. He was treated with posaconazole delayed-release tablet. This report describes the pharmacokinetic/pharmacodynamic study, safety profile, and determination of the appropriate dosage of posaconazole delayed-release tablet in a patient with coexisting invasive aspergillosis and mucormycosis. Posaconazole exposure was analyzed by noncompartmental model. Ratio of area under the plasma concentration-time curve over the minimum inhibitory concentration (AUC/MIC) was applied to maximize the efficacy of posaconazole. The loading dose of $300 \mathrm{mg} \mathrm{q} 12 \mathrm{hrs}$ was found to be potentially insufficient for achieving the AUC/MIC target for treatment of invasive mold infection with minimum inhibitory concentrations $>0.01 \mathrm{mg} / \mathrm{L}$. Early therapeutic drug monitoring to detect the drug concentration of posaconazole delayed-release tablet is necessary so that dosing adjustments can be made, as needed. In addition, a maintenance dose of either 400 or $300 \mathrm{mg}$ once daily could achieve the AUC/MIC targets. These maintenance dosing regimens effectuated a successful clinical outcome with minimal adverse events.
\end{abstract}

Keywords: antifungal, treatment, therapeutic drug monitoring, AUC/MIC, invasive fungal infection, mixed infection

\section{Introduction}

Invasive mold infections are life-threatening infections that are caused by highly virulent filamentous fungi that are angioinvasive, which results in tissue necrosis. ${ }^{1,2}$ The most common invasive mold infection of the lung is invasive pulmonary aspergillosis, followed by pulmonary mucormycosis. Several guidelines suggest early appropriate antifungal therapy to improve clinical outcomes and survival in patients with invasive mold infection. ${ }^{1-3}$ Dual infection with aspergillosis and mucormycosis in the same patient is extremely rare, and is an antifungal therapeutic challenge. Antifungal agents that currently exhibit activities against both Aspergillus and Mucorales include amphotericin B, isavuconazole, and posaconazole.

Posaconazole is a second-generation triazole with broad-spectrum antifungal activities. Posaconazole is currently available in the following three formulations: suspension, delayed-release tablet, and injection. Posaconazole delayed-release 
tablet was developed by mixing posaconazole with a $\mathrm{pH}-$ sensitive polymer via hot melt extrusion technology that allows the drug to release in the high-pH environment of the small intestine to enhance drug absorption, and limits its release in the stomach. ${ }^{4,5}$ As a result, the blood serum levels of posaconazole delayed-release tablet are less variable than those observed when suspension formulation is given. However, it is still necessary to monitor plasma posaconazole levels when delayed-release tablet is given to ensure that the levels are adequate to prevent or treat fungal infections. Therapeutic drug monitoring (TDM) of posaconazole is characterized by a concentration-effect relationship. Target level of posaconazole for prophylaxis is a trough concentration of $>0.7 \mathrm{mg} / \mathrm{L}$, which showed the lowest level of clinical failure. ${ }^{6}$ The target level for treatment of invasive fungal infection is a trough concentration of $>1.0 \mathrm{mg} / \mathrm{L}$ and an average concentration at steady state $\left(\mathrm{C}_{\mathrm{avg}}\right)$ of $>1.25 \mathrm{mg} / \mathrm{L}$, which was associated with a clinical response rate of $75 \% .^{7-9}$ However, the target posaconazole plasma concentration for treatment is derived from studies in patients with invasive aspergillosis, without correlation to the minimum inhibitory concentration (MIC) of the fungal pathogen. In in vivo and in vitro studies, the area under the plasma concentration-time curve from 0 to 24 hrs $\left(\mathrm{AUC}_{0-24}\right)$ over the $\mathrm{MIC}$ ratio (AUC/MIC) was found to be the best pharmacokinetic and pharmacodynamic (PK/PD) parameter for monitoring posaconazole treatment of invasive fungal infection. For aspergillosis, studies in neutropenic murine model showed AUC/MIC ratios of 167,309 , and 441 to be associated with $50 \%, 80 \%$, and $90 \%$ response relative to the reduction of galactomannan concentration, respectively. ${ }^{10,11}$ Similarly, an AUC/MIC ratio of 178 was required to achieve $50 \%$ response relative to suppression of the lung fungal burden in murine neutropenia model. ${ }^{11,12}$ In mucormycosis, an AUC/MIC of $>100$ was associated with a $90 \%$ response relative to suppression of lung fungal burden in murine neutropenic model. $^{13}$

There is currently no human study in the relationship between posaconazole AUC/MIC and the efficacy of treatment for coexisting aspergillosis and mucormycosis. However, the target $\mathrm{AUC} / \mathrm{MIC}$ ratio is much lower in mucormycosis than in aspergillosis. Posaconazole delayed-release tablet was approved in November 2013 by the US Food and Drug Administration (USFDA) for prophylaxis against invasive fungal infection. There is currently no data available regarding the recommended dose of delayed-release posaconazole tablet for the treatment of invasive fungal infection. The use of TDM would be beneficial for dose adjustment of posaconazole delayed-release tablet similar to the use of TDM for dose adjustment of posaconazole oral suspension. Here, we report the first case study of the use of pharmacokinetic/ pharmacodynamic relationship to identify the appropriate dosage of posaconazole delayed-release tablet for the treatment of coexisting invasive pulmonary aspergillosis and mucormycosis in an adult Thai patient based on the AUC/MIC target.

\section{Case report}

A 44-year-old previously healthy Thai man was admitted due to shortness of breath and respiratory failure following a car accident that resulted in his body becoming fully submerged in muddy water. He was treated as aspiration pneumonia with broad-spectrum antibiotic without improvement. He subsequently underwent chest computed tomography, which showed multiple air-filled cavities and intracavitary nodules in segmental consolidation at the apical, posterior segments of the right upper lobe, and scattered multi-focal consolidations in all segments of both lungs. He was intubated, and cultures from tracheal aspirate grew Aspergillus flavus, Aspergillus fumigatus, Rhizopus microsporus, and Lichtheimia corymbifera. The patient's blood galactomannan level was $>6.0$. Coexisting invasive pulmonary aspergillosis and mucormycosis was diagnosed. He was treated with liposomal amphotericin B (5 mg/kg/day) for 4 months. During therapy, the patient's serum creatinine increased from 0.9 to $1.46 \mathrm{mg}$ / $\mathrm{dL}$. In response, his treatment was switched to posaconazole delayed-release tablets. The patient received delayedrelease posaconazole $300 \mathrm{mg}$ twice daily on day 1 , and then $400 \mathrm{mg}$ once daily from day 2 to day 14 , and then $300 \mathrm{mg}$ once daily thereafter for 4 months.

Blood samples were collected for the loading doses on day 1 , and for the maintenance doses on days 7,14 , and 35 of posaconazole therapy. For the loading doses day 1 , blood samples were collected immediately prior to the first dose; at 2, 4, 6, 8, and $12 \mathrm{hrs}$ after administration of the first dose; and, at $12 \mathrm{hrs}$ after administration of the second dose. For the maintenance doses on days 7, 14, and 35, blood samples were collected at $0 \mathrm{hr}$ (pre-dose), and at 2, 4, $6,8,12$, and $24 \mathrm{hrs}$ after administration of posaconazole. Plasma posaconazole concentrations were analyzed by ACQUITY Ultra-Performance Liquid Chromatography with a photodiode array detector (Waters Corporation, Milford, MA, USA). This method was developed and 
fully validated according to USFDA guidelines. The pharmacokinetic parameters of posaconazole delayed-release tablet were analyzed by noncompartmental model using a pharmacokinetic software program (Winnonlin version 8.0; Pharsight Corporation, Sunnyvale, CA, USA).

Pharmacokinetic parameters of posaconazole delayed-release tablet on days $1,7,14$, and 35 in this patient are summarized in Table 1, and plasma concentrations are shown in Figure 1. On day 1 (loading dose), the minimum concentration $\left(\mathrm{C}_{\min }\right), \mathrm{AUC}_{0-\mathrm{t}}$, and $\mathrm{C}_{\mathrm{avg}}$ after the first dose were lower than those measured after subsequent dosages that were given on days 7,14 , and 35 (Table 1). In addition, time to $\mathrm{C}_{\max }\left(\mathrm{T}_{\max }\right)$ of posaconazole $300 \mathrm{mg}$ was lower than that of posaconazole $400 \mathrm{mg}\left(\mathrm{T}_{\max }=2\right.$ and $6 \mathrm{hrs}$, respectively). The posaconazole AUC/MICs for aspergillosis and mucormycosis in this patient are summarized in Table 2. The posaconazole AUC/MICs in this patient were lowest after the first dose of treatment.

On day 7 of posaconazole treatment, the patient had asymptomatic hypokalemia (serum potassium $=3.1 \mathrm{mEq} /$ L). Asymptomatic hypokalemia persisted despite reduction in posaconazole dose from $400 \mathrm{mg}$ once daily to $300 \mathrm{mg}$ once daily (serum potassium $=2.2-3.1 \mathrm{mEq} / \mathrm{L}$ and $2.5-3.3$ $\mathrm{mEq} / \mathrm{L}$, respectively). In response, the patient was treated with oral potassium chloride supplementation.

On day 35 of posaconazole treatment, the patient had no sign or symptom of recurrent invasive pulmonary aspergillosis or mucormycosis. Chest computed tomography showed marked improvement (Figure 2). Four months later, the patient returned to normal life and posaconazole was discontinued.

\section{Discussion}

To our knowledge, this is the first case report of PK/PD study to evaluate posaconazole delayed-release tablet for the treatment of a patient with coexisting invasive pulmonary aspergillosis and mucormycosis. The purpose of this report was to present the PK/PD and safety profile, and to investigate the appropriate dosage of posaconazole delayed-release tablet for the treatment of invasive fungal infection based on the PK/ PD relationship. The primary parameter of the PK/PD relationship for posaconazole was reported to be the AUC/MIC ratio. ${ }^{14}$ As such, our study used the AUC/MIC ratio as a major parameter in the $\mathrm{PK} / \mathrm{PD}$ relationship between posaconazole and fungal pathogens. The target AUC/MIC ratio was 300-500 for aspergillosis, and over 100 for mucormycosis according to the results previously observed in neutropenic murine models..$^{10-13}$ In addition, our study evaluated the pharmacokinetics of loading dose and maintenance dose of posaconazole delayed-release tablet by noncompartmental analysis. The results of our study revealed the $\mathrm{C}_{\min }$ and $\mathrm{C}_{\mathrm{avg}}$ of the loading dose and maintenance dose of posaconazole to be sufficient for achieving the target $C_{\min }(>1 \mathrm{mg} / \mathrm{L})$ and $\mathrm{C}_{\text {avg }}$ $(>1.25 \mathrm{mg} / \mathrm{L})$ for the treatment of aspergillosis. These findings are consistent with the results of previous

Table I Pharmacokinetic parameters on day I after loading dose of $300 \mathrm{mg}$ twice daily, on day 7 and day I4 after maintenance dose of $400 \mathrm{mg}$ once daily, and $300 \mathrm{mg}$ once daily on day 35

\begin{tabular}{|c|c|c|c|c|c|c|c|c|c|c|}
\hline Dose & Day & $\begin{array}{l}C_{\min } \\
(\mathrm{mg} / \mathrm{L})\end{array}$ & $\begin{array}{l}\text { AUC }_{0-\mathrm{t}} \\
(\mathrm{h} \mathrm{mg} / \mathrm{L})\end{array}$ & $\begin{array}{l}\mathrm{AUC}_{0-}{ }^{\infty} \\
(\mathrm{h} \mathrm{mg} / \mathrm{L})\end{array}$ & $\begin{array}{l}C_{\text {avg }} \\
(\mathrm{mg} / \mathrm{L})\end{array}$ & $\begin{array}{l}C_{\max } \\
(\mathrm{mg} / \mathrm{L})\end{array}$ & $\begin{array}{l}T_{\max } \\
\text { (hrs) }\end{array}$ & $\begin{array}{l}T_{1 / 2} \\
\text { (hrs) }\end{array}$ & $\begin{array}{l}\text { CL/F } \\
\text { (L/hrs) }\end{array}$ & $\begin{array}{l}\text { Vd/F } \\
\text { (L) }\end{array}$ \\
\hline $\begin{array}{l}300 \mathrm{mg} \\
\text { q } 12 \mathrm{hrs}\end{array}$ & I & $\begin{array}{l}0.57 \\
\text { (at } 12 \mathrm{hrs} \text { ) } \\
\text { I.78 } \\
\text { (at } 24 \mathrm{hrs} \text { ) }\end{array}$ & $\begin{array}{l}8.15 \\
\text { (at } 12 \text { hrs) } \\
16.30 \\
\text { (at } 24 \text { hrs) }\end{array}$ & 20.90 & $\begin{array}{l}0.68 \\
\text { (at } 12 \\
\text { hrs) } \\
1.36 \\
\text { (at } 24 \\
\text { hrs) }\end{array}$ & $\begin{array}{l}0.88 \\
\text { (at } 12 \mathrm{hrs} \text { ) }\end{array}$ & 2.0 & 15.42 & 14.35 & 319.70 \\
\hline $\begin{array}{l}400 \mathrm{mg} \\
\text { q } 24 \mathrm{hrs}\end{array}$ & 7 & 2.48 & 76.18 & 179.20 & 3.17 & 3.92 & 6.0 & 28.80 & 5.25 & 217.87 \\
\hline $\begin{array}{l}400 \mathrm{mg} \\
\text { q } 24 \mathrm{hrs}\end{array}$ & 14 & 2.59 & 77.46 & 201.28 & 3.23 & 3.81 & 6.0 & 33.16 & 5.169 & 247.08 \\
\hline $\begin{array}{l}300 \mathrm{mg} \\
\text { q } 24 \mathrm{hrs}\end{array}$ & 35 & 1.54 & 49.23 & 129.29 & 2.05 & 3.18 & 2.0 & 35.97 & 6.09 & 315.74 \\
\hline
\end{tabular}

Abbreviations: $C_{\min }$, minimum plasma concentration $\left(C_{\min }\right.$ on day I was determined at 12 hrs before next dose on day I; however, on days 7 , 14 , and 35 , the $C_{\min }$ was determined at $24 \mathrm{hrs}$ before next dose to be given the next day); $\mathrm{AUC}_{0-\mathrm{t}}$, area under concentration-time curve from time zero to time of the last quantifiable sample (time of the last quantifiable sample was $12 \mathrm{hrs}$ on day I, and $24 \mathrm{hrs}$ on days 7,14 , and 35); $A U C_{0-\infty}$, area under concentration-time curve from time zero to infinity; $C_{\text {avg, average }}$ concentration at steady state $\left(\mathrm{C}_{\text {avg }}=A \cup C_{0-t} /\right.$ dosing interval); $C_{\max }$, maximum plasma concentration; $T_{\max }$, time of maximum plasma concentration; $T_{1 / 2}$, terminal-phase halflife; $\mathrm{CL} / \mathrm{F}$, apparent total body clearance $\left(\mathrm{CL} / \mathrm{F}=\right.$ dose/ $\left.\mathrm{AUC}_{0-}{ }^{\infty}\right) ; \mathrm{Vd} / \mathrm{F}$, apparent volume of distribution. 


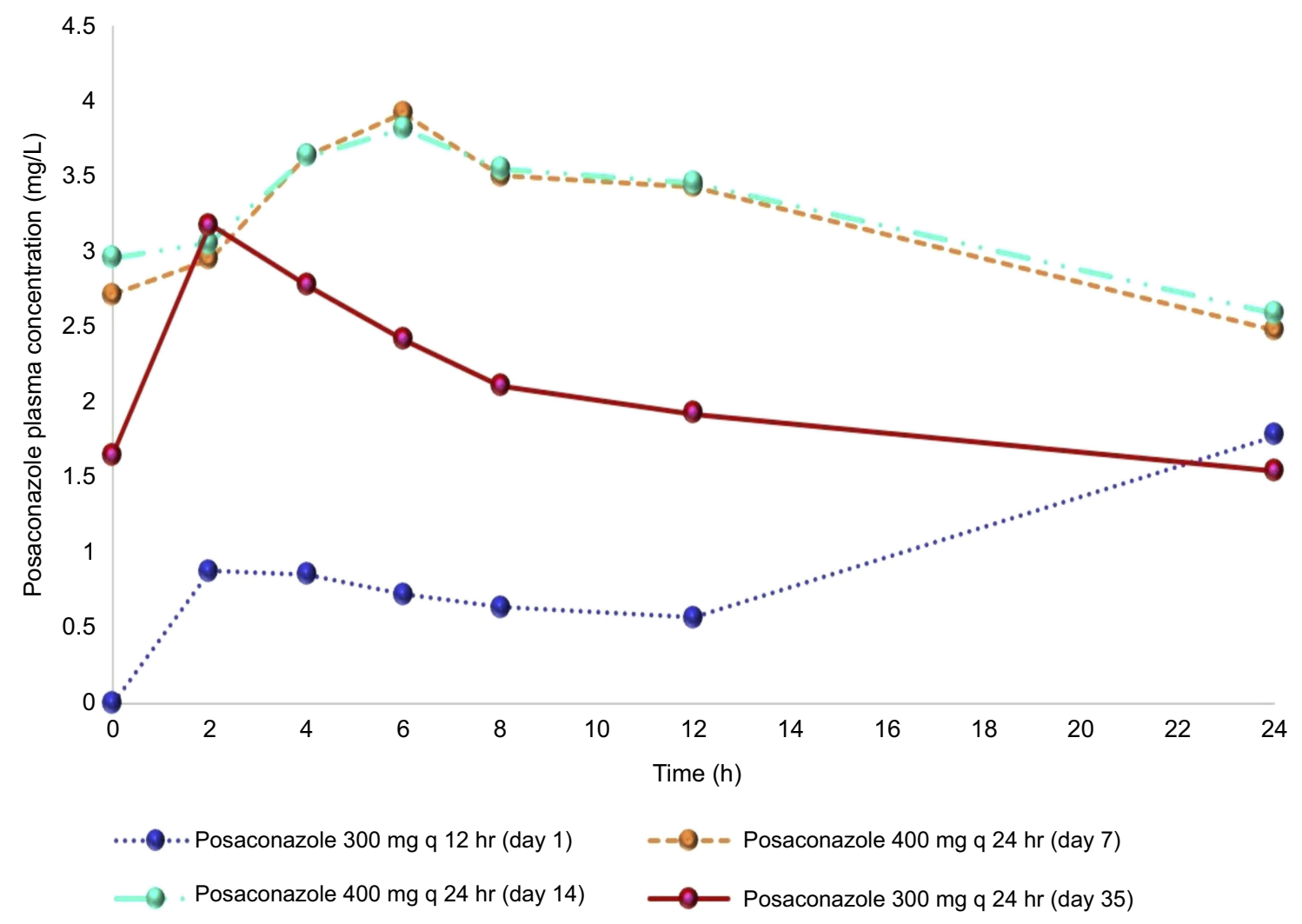

Figure I Posaconazole plasma concentration on days I, 7, I4, and 35 in a patient with coexisting invasive aspergillosis and mucormycosis.

Table 2 Posaconazole serum AUC/MIC target for aspergillosis and mucormycosis

\begin{tabular}{|c|c|c|c|c|c|c|c|c|}
\hline \multicolumn{2}{|c|}{ Fungal culture } & \multirow{2}{*}{$\begin{array}{l}\text { MIC of posa- } \\
\text { conazole } \\
(\mathrm{mg} / \mathrm{L})\end{array}$} & \multirow{2}{*}{$\begin{array}{l}\text { Target of } \\
\text { AUC/MIC } \\
\text { ratio }\end{array}$} & $\mathrm{AUC}_{0-12} I$ & \multicolumn{4}{|c|}{$\mathrm{AUC}_{0-24} / \mathrm{MIC}$ ratio } \\
\hline & & & & \multicolumn{2}{|c|}{$\begin{array}{l}300 \mathrm{mg} \mathrm{q} \mathrm{I} 2 \mathrm{hrs} \\
\text { (day I) }\end{array}$} & $\begin{array}{l}400 \mathrm{mg} \\
\text { q } 24 \mathrm{hrs} \\
\text { (day } 7 \text { ) }\end{array}$ & $\begin{array}{l}400 \mathrm{mg} \\
\text { q } 24 \text { hrs } \\
\text { (day I4) }\end{array}$ & $\begin{array}{l}300 \mathrm{mg} \\
\text { q } 24 \mathrm{hrs} \\
\text { (day 35) }\end{array}$ \\
\hline \multirow[t]{2}{*}{ Aspergillosis } & A. fumigatus & 0.01 & $300-500$ & 815.15 & $1,630.30$ & $7,618.43$ & $7,746.44$ & $4,923.02$ \\
\hline & A. flavus & 0.12 & $300-500$ & 67.93 & 135.86 & 634.87 & 645.54 & 410.25 \\
\hline \multirow[t]{2}{*}{ Mucormycosis } & R. microsporus & 0.25 & $>100$ & 32.61 & 65.21 & 304.74 & 309.86 & 196.92 \\
\hline & L. corymbifera & 0.25 & $>100$ & 32.61 & 65.21 & 304.74 & 309.86 & 196.92 \\
\hline
\end{tabular}

Abbreviations: MIC, minimum fungicidal inhibitory concentration; AUC/MIC ratio, area under concentration-time curve over minimum fungicidal inhibitory concentration $(\mathrm{MIC})$; $\mathrm{AUC}_{0-12}$, area under concentration-time curve from 0 to $12 \mathrm{hrs}$; $\mathrm{AUC}_{0-24}$, area under concentration-time curve from 0 to 24 hrs.

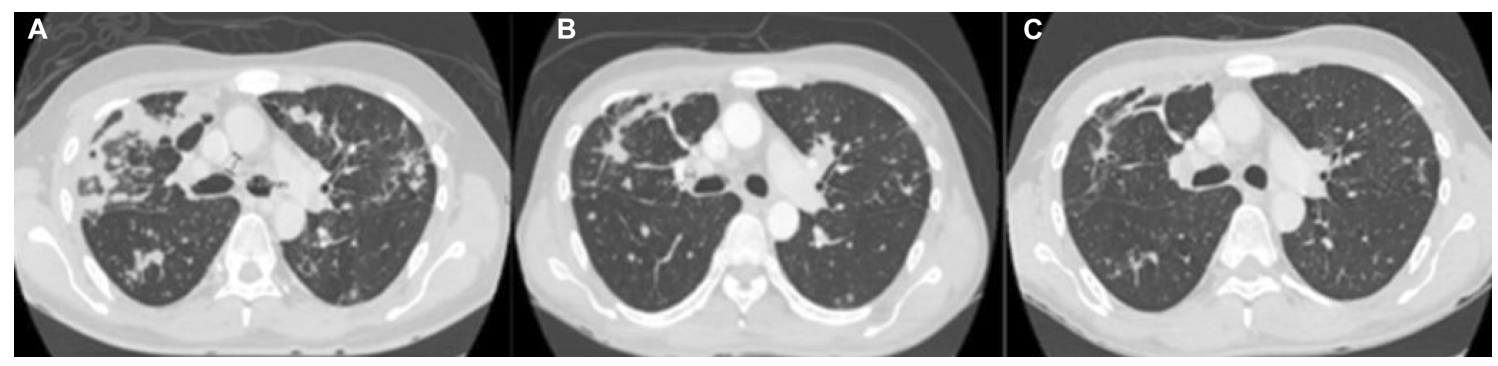

Figure 2 Chest computed tomography of a patient with invasive pulmonary aspergillosis and mucormycosis. Before the start of posaconazole (A); day 35 after the start of posaconazole treatment (B); and, day 90 after the start of posaconazole treatment (C). 
pharmacokinetic studies. ${ }^{15-17}$ Interestingly, our study showed the $\mathrm{T}_{\max }$ of posaconazole $400 \mathrm{mg}$ ( $6 \mathrm{hrs}$ to be longer than that of $300 \mathrm{mg}$ ( $2 \mathrm{hrs}$ ). These findings may be explained by a saturation of the absorption process and the formulation of posaconazole delayed-release tablet. Posaconazole has high lipophilicity and low aqueous solubility, which is the cause of its dose-limited absorption. ${ }^{18}$ The delayed-release formulation increases the dissolution of posaconazole, which enhances posaconazole exposure Therefore, this potential existence of a saturation of the absorption process may be the cause of the observed dose-dependent $\mathrm{T}_{\max }$. We found the AUC of the loading dose and the maintenance dose of posaconazole delayed-release tablet to be comparable with those reported from previous studies. ${ }^{15-17}$ The $\mathrm{AUC}_{0-12}$ of the loading dose of posaconazole delayed-release tablet (300 mg q $12 \mathrm{hrs}$ ) in this study and a previous study was 8.15 and $6.24 \pm 1.75 \mathrm{~h} \cdot \mathrm{mg} / \mathrm{L}$, respectively. ${ }^{16}$ In contrast, when compared with posaconazole oral suspension $400 \mathrm{mg}$ single dose and multiple dose (400 mg q 12 hrs and $200 \mathrm{mg} \mathrm{q} 6$ hrs), the AUCs of posaconazole oral suspension were lower than that of posaconazole delayed-release tablet shown in our study. ${ }^{19}$ These findings may be explained by the delayedrelease formulation, which may confer more benefit in invasive mold infection than that delivered by oral suspension.

In addition, the patient's plasma posaconazole concentration was lower on day 1 than on the subsequent days, which suggests that the loading dose may have been insufficient to reach the AUC/MIC target. The result of this study showed that only Aspergillus fumigatus achieved the AUC/MIC target of 300-500 after loading dose of $300 \mathrm{mg} \mathrm{q} 12 \mathrm{hrs}$, because it had a low MIC (MIC $0.01 \mathrm{mg} / \mathrm{L})$. In contrast, Aspergillus flavus, Rhizopus microsporus, and Lichtheimia corymbifera all had higher MICs $(0.12,0.25$, and $0.25 \mathrm{mg} / \mathrm{L}$, respectively). Therefore, posaconazole delayed-release tablet at a loading dose of $300 \mathrm{mg}$ q 12 hrs may have been insufficient to treat the Aspergillus spp. and Mucorales that had high MICs in our patient. This finding is consistent with a previous population pharmacokinetic study that found that approximately $72.6 \%$ of patients achieved the posaconazole minimum concentration target after 48 hrs of treatment. ${ }^{20}$ This may be due to the inter-individual variability and inter-occasion variability pharmacokinetic characteristics of posaconazole. ${ }^{20}$ These results further support the necessity for TDM in posaconazole delayed-release tablet. In our patient, an increase in the dose or frequency of posaconazole at loading dose was required to achieve the AUC/ MIC target. Patients with coinfection (like our patient), patients that are critically ill, and patients that are immunocompromised are at increased risk of having interindividual pharmacokinetic variability. Moreover, these patients have increased clearance and/or distribution volume, which affects the pharmacokinetics of posaconazole. To date, no human studies in the AUC/MIC target have been published. Our study used data from a previous study in which the AUCs of single-dose posaconazole tablets of 800 and $1,200 \mathrm{mg}$ were similar (AUC $=49.84$ \pm 21.43 and $45.26 \pm 19.91 \mathrm{~h} \cdot \mathrm{mg} / \mathrm{L}$, respectively). ${ }^{18}$ It is possible that the absorption of single-dose posaconazole was saturated at doses $>800 \mathrm{mg} .{ }^{18}$ Thus, alternative dosing strategies may be used by increasing the loading dose of posaconazole delayed-release tablet from $300 \mathrm{mg}$ q $12 \mathrm{hrs}$ to either $600 \mathrm{mg} \mathrm{q} 12 \mathrm{hrs}$ or $300 \mathrm{mg} \mathrm{q} 6 \mathrm{hrs}$. This would increase the AUC by twofold and achieve the AUC/MIC target for both aspergillosis and mucormycosis. Importantly, the safety of a higher dose than $300 \mathrm{mg}$ q $12 \mathrm{hrs}$ of posaconazole should be monitored.

For maintenance dose regimen, our study found the AUC of posaconazole $300 \mathrm{mg}$ once daily and $400 \mathrm{mg}$ once daily to be three- to fourfold higher than the loading dose at $300 \mathrm{mg} \mathrm{q} 12 \mathrm{hrs}$, and that they achieved the AUC/ MIC target similar to the findings of previous neutropenic murine model studies. ${ }^{10-13}$ Thus, maintenance dose of posaconazole delayed-release tablet $300 \mathrm{mg}$ per day reached the AUC/MIC target of 300-500 for aspergillosis when the maximum MICs were $\leq 0.10 \mathrm{mg} / \mathrm{L}$. Furthermore, if the maintenance dose of posaconazole increases from $300 \mathrm{mg}$ per day to $400 \mathrm{mg}$ per day, the AUC/MIC target would be achieved for a MIC up to $0.15 \mathrm{mg} / \mathrm{L}$. For mucormycosis, which requires an AUC/MIC target of $>100$, a maintenance dose of posaconazole $300 \mathrm{mg}$ per day will achieve the target when the MIC is<$0.50 \mathrm{mg} / \mathrm{L}$. Mucormycosis with a higher MIC within the range of $0.50-0.75 \mathrm{mg} / \mathrm{L}$ requires an increase in posaconazole dose up to $400 \mathrm{mg}$ per day to achieve the AUC/ MIC target. Accordingly, the AUC/MIC ratio for posaconazole may be an additional tool for monitoring treatment outcomes of mucormycosis and aspergillosis.

Asymptomatic hypokalemia is a safety concern in patients receiving posaconazole. In the previous study, the incidence of hypokalemia in posaconazole delayed-release tablet was $10 \%$ at a dose of $200 \mathrm{mg}$ per day, and $22 \%$ at a dose of $300 \mathrm{mg}$ per day. ${ }^{17,18}$ Our study found no association between hypokalemia and plasma posaconazole concentration. This finding was also observed in posaconazole suspensions. ${ }^{8}$ This may be partially explained by the fact that concentrations of 
posaconazole in humans are quite variable. In addition, two cases of symptomatic hypokalemia were reported in one adolescent patient and one adult patient who received posaconazole delayed-release tablet with high plasma posaconazole concentration (9.7 and 5.18-7.98 mg/L, respectively). ${ }^{21,22}$ Interestingly, it was found in the latter case that hypokalemia was related to high plasma posaconazole concentrations, and the hypokalemia resolved when plasma posaconazole concentration decreased to $2.16 \mathrm{mg} / \mathrm{L}{ }^{22}$ Therefore, both high-dose and low-dose posaconazole delayed-release tablet can cause hypokalemia, which highlights the importance of regular monitoring of serum potassium levels. The mechanism of posaconazole-induced hypokalemia is not yet known, but it may be related to mineralocorticoid effect. In murine model study, posaconazole interfered with the mineralocorticoid pathway by inhibiting 11b-hydroxysteroid dehydrogenase type 2 , which resulted in a loss of potassium via urine. ${ }^{23}$ Another triazole that showed mineralocorticoid effect was itraconazole. Posaconazole was developed from itraconazole, so it may have the same effects. This case report has some mentionable limitations. First, only one patient was included, so it was not possible to determine factors that affect the pharmacokinetics. Second and last, the patient received liposomal amphotericin B prior to switching to posaconazole, and this may have impacted the clinical outcome as well as hypokalemia in this study.s

\section{Conclusion}

Posaconazole delayed-release tablet demonstrated higher AUC and less pharmacokinetic variability than oral suspension formulation. In accordance with the PK/PD parameter, posaconazole delayed-release tablet at $300 \mathrm{mg} \mathrm{q} 12$ hrs on day 1 showed an AUC that was insufficient for eradicating molds with high MICs. Therefore, it may be necessary to increase the loading dose to achieve the AUC/MIC target on the first day. Early TDM is necessary for dosing adjustment in similarly complicated cases. Posaconazole delayed-release tablet at a maintenance dose of 300 or $400 \mathrm{mg}$ once daily may be effective and safe for treating coexisting invasive pulmonary aspergillosis and mucormycosis; however, these results reflect data from only one patient, so further studies are needed to verify the efficacy of this regimen.

\section{Ethical issues}

This study was approved by Siriraj Institutional Review Board (SiRB) (COA no. Si645/2016), and the patient provided written informed consent approving that the details of his case be published.

\section{Acknowledgments}

The authors gratefully acknowledge the patient profiled in this report for allowing us to disclose details relating to his case, and the Faculty of Medicine Siriraj Hospital, Mahidol University for permitting us to conduct a pharmacokinetic/ pharmacodynamic study of posaconazole delayed-release tablet in this case of coexisting invasive pulmonary aspergillosis and mucormycosis. Dr Methee Chayakulkeeree was supported by a Chalermphrakiat Grant from the Faculty of Medicine Siriraj Hospital, Mahidol University, Bangkok, Thailand.

\section{Disclosure}

All authors declare no personal or professional conflicts of interest, and no financial support from the companies that produce and/or distribute the drugs, devices, or materials described in this report.

\section{References}

1. Cornely OA, Arikan-Akdagli S, Dannaoui E, et al. ESCMID and ECMM joint clinical guidelines for the diagnosis and management of mucormycosis 2013. Clin Microbiol Infect. 2014;20(Suppl 3):5-26. doi:10.1111/1469-0691.12371

2. Patterson TF, Thompson GR 3rd, Denning DW, et al. Practice guidelines for the diagnosis and management of aspergillosis: 2016 update by the Infectious Diseases Society of America. Clin Infect Dis. 2016;63(4):e1-e60. doi:10.1093/cid/ciw326

3. Tissot F, Agrawal S, Pagano L, et al. ECIL-6 guidelines for the treatment of invasive candidiasis, aspergillosis and mucormycosis in leukemia and hematopoietic stem cell transplant patients. Haematologica. 2017;102 (3):433-444. doi:10.3324/haematol.2016.152900

4. Fule R, Amin P. Hot melt extruded amorphous solid dispersion of posaconazole with improved bioavailability: investigating drug-polymer miscibility with advanced characterisation. Biomed Res Int. 2014;2014:146781. doi:10.1155/2014/146781

5. Wiederhold NP. Pharmacokinetics and safety of posaconazole delayed-release tablets for invasive fungal infections. Clin Pharmacol. 2016;8:1-8. doi:10.2147/CPAA.S60933

6. Dolton MJ, Ray JE, Chen SC, Ng K, Pont L, McLachlan AJ. Multicenter study of posaconazole therapeutic drug monitoring: exposure-response relationship and factors affecting concentration. Antimicrob Agents Chemother. 2012;56:5503-5510. doi:10.1128/ AAC.00802-12

7. Ashbee HR, Barnes RA, Johnson EM, Richardson MD, Gorton R, Hope WW. Therapeutic drug monitoring (TDM) of antifungal agents: guidelines from the British Society for Medical Mycology. J Antimicrob Chemother. 2014;69(5):1162-1176. doi:10.1093/jac/dkt508

8. Jang SH, Colangelo PM, Gobburu JV. Exposure-response of posaconazole used for prophylaxis against invasive fungal infections: evaluating the need to adjust doses based on drug concentrations in plasma. Clin Pharmacol Ther. 2010;88(1):115-119. doi:10.1038/clpt.2010.64

9. Ullmann AJ, Aguado JM, Arikan-Akdagli S, et al. Diagnosis and management of Aspergillus diseases: executive summary of the 2017 ESCMID-ECMM-ERS guideline. Clin Microbiol Infect. 2018;24 (Suppl 1):e1-e38. doi:10.1016/j.cmi.2018.01.002 
10. Howard SJ, Lestner JM, Sharp A, et al. Pharmacokinetics and pharmacodynamics of posaconazole for invasive pulmonary aspergillosis: clinical implications for antifungal therapy. J Infect Dis. 2011;203 (9):1324-1332. doi:10.1093/infdis/jir023

11. European Committee on Antimicrobial Susceptibility Testing [homepage on the Internet]. Posaconazole: rationale for the EUCAST clinical breakpoints, version $2.0 ; 2017$. Available from: http://www.eucast.org/ast_of fungi/rationale_documents_for_antifungals/. Accessed June 3, 2017.

12. Lepak AJ, Marchillo K, Vanhecker J, Andes DR. Posaconazole pharmacodynamic target determination against wild-type and Cyp51 mutant isolates of Aspergillus fumigatus in an in vivo model of invasive pulmonary aspergillosis. Antimicrob Agents Chemother. 2013;57(1):579-585. doi:10.1128/AAC.01279-12

13. Lewis RE, Albert ND, Kontoyiannis DP. Comparative pharmacodynamics of posaconazole in neutropenic murine models of invasive pulmonary aspergillosis and mucormycosis. Antimicrob Agents Chemother. 2014;58 (11):6767-6772. doi:10.1128/AAC.03569-14

14. Dekkers BGJ, Bakker M, van der Elst KCM, et al. Therapeutic drug monitoring of posaconazole: an update. Curr Fungal Infect Rep. 2016;10(51-61). doi:10.1007/s12281-016-0255-4.

15. Krishna G, Ma L, Martinho M, Preston RA, O`Mara E. A new solid oral tablet formulation of posaconazole: a randomized clinical trial to investigate rising single- and multiple-dose pharmacokinetics and safety in healthy volunteers. J Antimicrob Chemother. 2012;67 (11):2725-2730. doi:10.1093/jac/dks268

16. Duarte RF, Lopez-Jimenez J, Cornely OA, et al. Phase $1 \mathrm{~b}$ study of new posaconazole tablet for prevention of invasive fungal infections in high-risk patients with neutropenia. Antimicrob Agents Chemother. 2014;58(10):5758-5765. doi:10.1128/AAC.03050-14
17. Cornely OA, Duarte RF, Haider S, et al. Phase 3 pharmacokinetics and safety study of a posaconazole tablet formulation in patients at risk for invasive fungal disease. $J$ Antimicrob Chemother. 2016;71 (3):718-726. doi: $10.1093 / \mathrm{jac} / \mathrm{dkv} 380$

18. Courtney R, Pai S, Laughlin M, Lim J, Batra V. Pharmacokinetics, safety, and tolerability of oral posaconazole administered in single and multiple doses in healthy adults. Antimicrob Agents Chemother. 2003;47(9):2788-2795.

19. Krishna G, Moton A, Ma L, Medlock MM, McLeod J. Pharmacokinetics and absorption of posaconazole oral suspension under various gastric conditions in healthy volunteers. Antimicrob Agents Chemother. 2009;53(3):958-966. doi:10.1128/AAC.01034-08

20. Petitcollin A, Boglione-Kerrien C, Tron C, et al. Population pharmacokinetics of posaconazole tablets and monte carlo simulations to determine whether all patients should receive the same dose. Antimicrob Agents Chemother. 2017;61(11):e01166-17. doi:10.1128/AAC.01166-17

21. Martino J, Fisher BT, Bosse KR, Bagatell R. Suspected posaconazole toxicity in a pediatric oncology patient. Pediatr Blood Cancer. 2015;62(9):1682. doi:10.1002/pbc.25568

22. Mahmood M, Abu Saleh O, Sohail MR. Hypokalemia and hypertension associated with supratherapeutic posaconazole levels. Antimicrob Agents Chemother. 2017;61:4. doi:10.1128/AAC.00019-17

23. Beck KR, Bachler $M$, Vuorinen $A$, et al. Inhibition of 11beta-hydroxysteroid dehydrogenase 2 by the fungicides itraconazole and posaconazole. Biochem Pharmacol. 2017;130:93-103. doi:10.1016/j.bcp.2017.01.010
Therapeutics and Clinical Risk Management

\section{Publish your work in this journal}

Therapeutics and Clinical Risk Management is an international, peerreviewed journal of clinical therapeutics and risk management, focusing on concise rapid reporting of clinical studies in all therapeutic areas, outcomes, safety, and programs for the effective, safe, and sustained use of medicines. This journal is indexed on PubMed Central, CAS,

\section{Dovepress}

EMBase, Scopus and the Elsevier Bibliographic databases. The manuscript management system is completely online and includes a very quick and fair peer-review system, which is all easy to use. Visit http://www.dovepress.com/testimonials.php to read real quotes from published authors. 